Planta (1990) 181:475-486

\title{
Local motion and conformational changes in the cuticle of Clivia miniata Regel
}

\section{A microfluorescence and spin-label study}

\author{
A.P. Wunderlich ${ }^{1}$, H. Gaub ${ }^{1}$, D. Marsh ${ }^{2}$, and E. Sackmann ${ }^{1 *}$ \\ 1 Physik Department (Biophysics Group), Technische Universität München, D-8046 Garching, and \\ ${ }^{2}$ Max-Planck-Institut für Biophysikalische Chemie, Am Faßberg, D-3400 Göttingen, Federal Republic of Germany
}

\begin{abstract}
The temperature dependence of the local diffusion of fluorescent molecular probes of various polarities (alkane, long-chain fatty acid, short-chain alcohol and fatty acid), all labelled with 7-nitrobenz-2-oxa-1,3-diazol4-yl in the cuticle of Clivia miniata Regel was studied by the technique of fluorescence recovery after photobleaching. The technique yields the coefficient of diffusion, $D$, in the plane of the cuticle over distances of some $10 \mu \mathrm{m}$ and the fraction, $R$, of mobile reporter molecules. The inner (more hydrophilic) and the outer (more hydrophobic) faces of the cuticle were studied separately by appropriate incubation. The value of $D$ was found to depend sensitively on the polarity of the probe, the temperature and the position within the cuticle (outer hydrophobic or inner hydrophilic side). Depending on the type of probe, $D$ increased (in the case of the alkane) or decreased (in the case of the alcohol) after removal of the (monomeric) waxes. The electron-spin-resonance (ESR) spectra of incorporated spin-labelled fatty-acid probes measured in the intact cuticle contained a major component similar to the spectrum recorded from the polymerized matrix from which waxes had been extracted, and a second component similar to the spectrum from the monomeric waxes. At low temperatures, the ESR spectra from labels at two different chain positions corresponded to chain motion which was slow on the ESR timescale. At high temperatures, the spectral component from the monomeric waxes indicated chain motions in the motional narrowing regime which were of an essentially isotropic nature.

No evidence was found for a liquid-crystalline lipid phase such as found for the polar lipids in cell membranes, nor was there evidence for a sharp, thermotropic, lipid-phase transition either in the cuticle or in the waxes. Experiments with oriented samples did not demonstrate
\end{abstract}

* To whom correspondence should be addressed

Abbreviations: MX-membrane $=$ polymer matrix membrane (or monomeric wax-depleted cuticle); $\mathrm{ESR}=$ electron-spin resonance $n$-SASL $=n$-(4,4-dimethyl-N-oxy-2-oxazolidinyl)-stearic $\quad$ acid NBD = 7-nitrobenz-2-oxa-1,3-diazol-4-yl the presence of large domains with a uniform orientation of the lipid chains relative to the cuticular layers. The diffusion measurements and spin-label studies provide evidence for conformational changes of the cuticle extending over the whole temperature range studied $\left(10^{\circ} \mathrm{C}\right.$ to $70^{\circ} \mathrm{C}$ ). These conformational changes are attributed to phase-separation processes within the cuticle. The phase separation in extracted waxes extended over a similar broad temperature range. This indicates that the transitions in the cuticle are largely determined by these components. At higher temperatures, however, the chain mobility in the regions of monomeric wax was considerably greater than that in the polymerized matrix. The experimental results strongly indicate that all three layers of the Clivia cuticle exhibit a multilamellar structure of alternatingly stacked, highly hydrophobic layers of welldefined thickness $(5 \pm 0.5 \mathrm{~nm})$ and more disordered layers of variable ( 4 to $15 \mathrm{~nm}$ ) thickness. The lamellae are wellordered and extend over the whole leaf in the cuticle proper but are split-up into small domains in the inner and the external cuticular layer. Furthermore, changes of the molecular transport properties caused by the influence of ozone exerted during the growth of the plant were studied. We found that the diffusion coefficient increased both in the outer and the inner layer of the cuticle. A particularly large increase, by about a factor of three, was found for alkane diffusion in the hydrophobic outer face, pointing to defects in the polymerized matrix.

Key words: Clivia - Cuticle (conformational transition) - Ozone treatment - Photobleaching - Polymerized multilayer (cuticle) - Spin label

\section{Introduction}

The cuticle of plant leaves acts both as a protective layer against mechanical damage and as a selective barrier by which plants can control water exchange with the environment (Schmidt and Schönherr 1982). Moreover, 
it controls the penetration of herbicides, fertilizers and hazardous chemicals of various polarities (Juniper 1957; Kerler et al. 1984). It is also a strong barrier against attacks by microbes.

In order to serve both as mechanical protection and diffusion barrier the cuticle is designed as a composite material of polymerized cutin and monomeric waxes. However, pronounced birefringence shows that the cuticle is well organized on a micrometer scale (Sitte and Rennier 1963). The cuticle is a multicomponent system and is highly asymmetric with respect to the distribution of components and the molecular design between the inside and the outside of the leaflet. It can adapt its permeability to foreign molecules to changes in the environmental conditions (such as temperature or humidity) by variation of the (monomeric) wax composition (Baker 1974; Hallam 1980).

In the present work, we studied the local mobilities and structural features of the cuticle of Clivia miniata as well as modifications of these properties by the exposure of the plant to ozone during growth. Clivia cuticle is used as a model system for the present study since its outer layer exhibits a highly organized multilamellar structure. Moreover, the preparation of cuticle is easy.

Firstly, we report measurements of the local lateral diffusion coefficients of fluorescent probes of various polarities as a function of temperature. In particular, separate measurements are performed on both the inner and the outer side of the cuticular leaflet by selective incorporation of fluorescence dyes into one of these sides.

Secondly, we have studied the rotational dynamics and ordering of the lipid chains in the cuticle, using electron-spin-resonance (ESR) spectroscopy of incorporated fatty-acid probes spin-labelled at different positions in the chain. These results have been compared with the temperature dependence of the ESR spectra from the corresponding probes both in the extracted waxes and in the polymerized matrix freed of the waxes (MX-membrane, short for polymer matrix membrane).

\section{Material and experimental procedures}

Fluorescent labels. The following 7-nitrobenz-2-oxa-1,3-diazol-4-yl (NBD)-labelled molecules ( $\mathrm{I} a-\mathrm{d}$ of various polarities were used (Molecular Probes, Eugene, Ore., USA). The molecules are:<smiles>[R1]N(CCCCCCCC)CCCCCCCC</smiles>

NBD-dioctylamine $(\mathrm{MW}=404)$<smiles>[R]N([Tl])CCO</smiles>

NBD-methylaminoethanol $(\mathrm{MW}=239)$

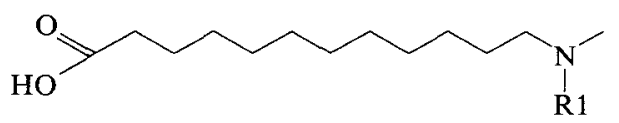

NBD-methylaminododecanoic acid (MW $=392)$<smiles>[R1]NCCCCCC(=O)O</smiles>

NBD-aminohexanoic acid $(\mathrm{MW}=294)$<smiles>[H][R]c1cc([N+](=O)[O-])c2nonc2c1C</smiles>

R1 denotes the fluorescent NBD group.

Spin probes. The spin probes were prepared according ot the method described by Hubbell and McConnell (1971).

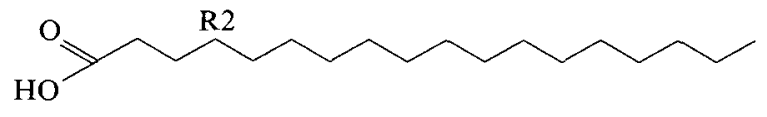

4-stearic acid spin label (4-SAS; $\mathrm{MW}=384)$

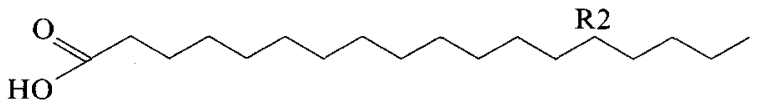

14-stearic acid spin label (14-SASL ; MW = 384)<smiles>[R2][CH]C1(C([2H])([2H])[2H])OCN1C</smiles>

R2 denotes the spin-label nitroxyl group.

Preparation of cuticles. Plants (Clivia miniata Regel; Gartencenter Seebauer, München, FRG) were grown in our laboratory at room temperature or in a climatic chamber at the institute for plant pathology of the Gesellschaft für Strahlen- und Umweltforschung (Neuherberg/Munich, FRG) in a natural or in an ozone-enriched (300-800 $\mu \mathrm{g} \cdot \mathrm{m}^{-3}$ ozone) atmosphere, respectively. In the chamber the plants were exposed to a light flux of $30000 \mathrm{~lx}$ during the day at a relative humidity of $70 \%$ and a temperature of $23-25^{\circ} \mathrm{C}$. At night the temperature was $15-18^{\circ} \mathrm{C}$ and the humidity $70-80 \%$. The cuticular leaflets from the adaxial side (stomata-free) of the leaves were prepared following the procedure of Orgell (Orgell 1955) by detachment from the pectin layer with pectinase. Pieces $\left(2 \cdot 2 \mathrm{~cm}^{2}\right)$ were cut from the leaves. In order to compare the data of the ozone-treated plants and the controls, all samples of cuticle were taken from fully expanded leaves $(35-45 \mathrm{~cm}$ in length) and from the same position on the leaf $(20-25 \mathrm{~cm}$ above leaf base). They were floated with the adaxial side uppermost on a $3 \%(\mathrm{v} / \mathrm{w})$ of pectinase (Roth, Karlsruhe, FRG) in $0.02 \mathrm{M}$ citric acid and $0.002 \mathrm{M} \mathrm{NaN}_{3}$ which was adjusted to $\mathrm{pH} 3$ using $\mathrm{NaOH}$. After incubation for $14 \mathrm{~d}$ the cuticular leaflet was removed from the cell fragments using forceps, and wahsed in $\mathrm{H}_{2} \mathrm{O}$ to remove residual tissue. In order to equilibrate the sample, measurements were performed at least $2 \mathrm{~d}$ after preparation. To remove the waxes, the isolated cuticular leaflets were incubated in sequence in methanol $(3 \times 10 \mathrm{~min}$ in fresh solvent), in chloroform/methanol $(1: 1, \mathrm{v} / \mathrm{v})$ $(3 \times 10 \mathrm{~min})$, and chloroform $(5 \times 10 \mathrm{~min})$. The chloroform was removed by washing first with chloroform/methanol, then with methanol and finally with water.

Spin-label studies. The fatty-acid spin probes were incorporated in the cuticular leaflets by incubation with an aqueous dispersion 
of the desired probe in $0.1 \mathrm{M} \mathrm{Na}_{2} \mathrm{CO}_{3} / \mathrm{NaHCO}_{3}$ buffer $\mathrm{pH} 9$. In order to incorporate the probes into the inner or outer side of the cuticle separately, a leaflet of size $2 \cdot 2^{2} \mathrm{~cm}$ was placed on a glass slide and the outer rim sealed with silicone grease to prevent access to the undersite. Drops of the aqueous spin-label dispersion were then deposited on the exposed side and subsequently washed off after a 5-min incubation at room temperature. The leaflet was finally trimmed from the slide and transferred to a flat quartz tissue cell (oriented sample). For the unoriented sample, leaflets were folded to avoid contact between the opposite faces of the cuticle, and sealed into a glass capillary ( $1 \mathrm{~mm}$ i.d.). Sufficient buffer was included in the sample cells to ensure complete hydration throughout the range of temperatures studied. The extracted monomeric waxes were spin-labelled by addition of spin label in chloroform solution, and the chloroform was then removed by evaporation in a stream of nitrogen, either on the flat surface of the quartz tissue cell or in a round-bottomed tube. In the latter case, the dry wax was then transferred and sealed in a glass capillary $(1 \mathrm{~mm}$ i.d.).

Electron-spin-resonance spectra were recorded on a Varian E-line or a Century-line ESR-spectrometer (Varian, Palo Alto, Cali., USA) operating at a frequency of $9 \mathrm{GHz}$. For measurements with unoriented samples the capillaries were accommodated within 4-mm-diameter quartz tubes containing light silicone oil for thermal stability. The microwave cavity was mounted vertically and the temperature controlled by nitrogen gas flow with a double-wall quartz dewar. For measurements with oriented samples, the cavity was mounted horizontally and the orientation of the sample relative to the spectrometer field was determined using a specially constructed vernier goniometer. The entire cavity was thermostatted by nitrogen gas flow. Sample temperature was measured by a finewire thermocouple dipping into the silicone oil at the top of the microwave cavity, or attached directly to the flat tissue cell. Continuous temperature scans were performed by locking the spectrometer to the central ESR peak by feeding the second derivative signal to the field-frequency lock unit. The line height was then displayed against the linearized thermocouple output on an $x-y$ recorder.

Lateral-diffusion measurement by the technique of fuorescence recovery after photobleaching (FRAP). The fluorescent dyes Ib to Id were incorporated into the cuticle by application of aqueous solutions of these dyes. The water-insoluble dye Ia was incorporated into vesicles of dimyristoylphosphatidyl-choline, in order to avoid carriers like dimethylsulfoxide (DMSO) which might damage the structure of the cuticle, and the vesicle suspension applied to the cuticle.

The separate incorporations into the outer and the innermost layers of the cuticular leaflets were done as described for the spin probes. Incubation times were of the order of about $1 \mathrm{~h}$. The highly hydrophilic dye I c (NBD-aminohexanoic acid) can only be incorporated into the hydrophilic side. The cuticles were left on the glass plates for the FRAP experiment.

The photobleaching experiments were performed with a FRAP apparatus built around a Zeiss Axiomat microscope (Gaub et al. 1984). The lateral diffusion coefficients were measured by the spotbleaching technique. The beam of an argon laser was divided into a bleaching beam and an observation beam with the help of two semitransparent mirrors of $10 \%$ reflectivity. The intensity of the attenuated observation beam was smaller by a factor of $10^{-3}$ than the non-attenuated bleaching beam. Both beams were focussed to the same spot on the surface of the cuticle by an oil-immersion objective (Planapo PH3 100x oil; Zeiss, Oberkochen, FRG). This allowed a small area (diameter $6 \mu \mathrm{m}$ ) to be illuminated. A computer-controlled shutter allowed the observer to change rapidly between the two beams.

The light emitted from the bleached area of the cuticle was passed through a set of interference filters and a dichroitic mirror to ensure proper separation of excitation and emission light. The fluorescence light intensity was measured by a photomultiplier tube (RCA 31034-04). Alternatively, the probe can be observed by bino-
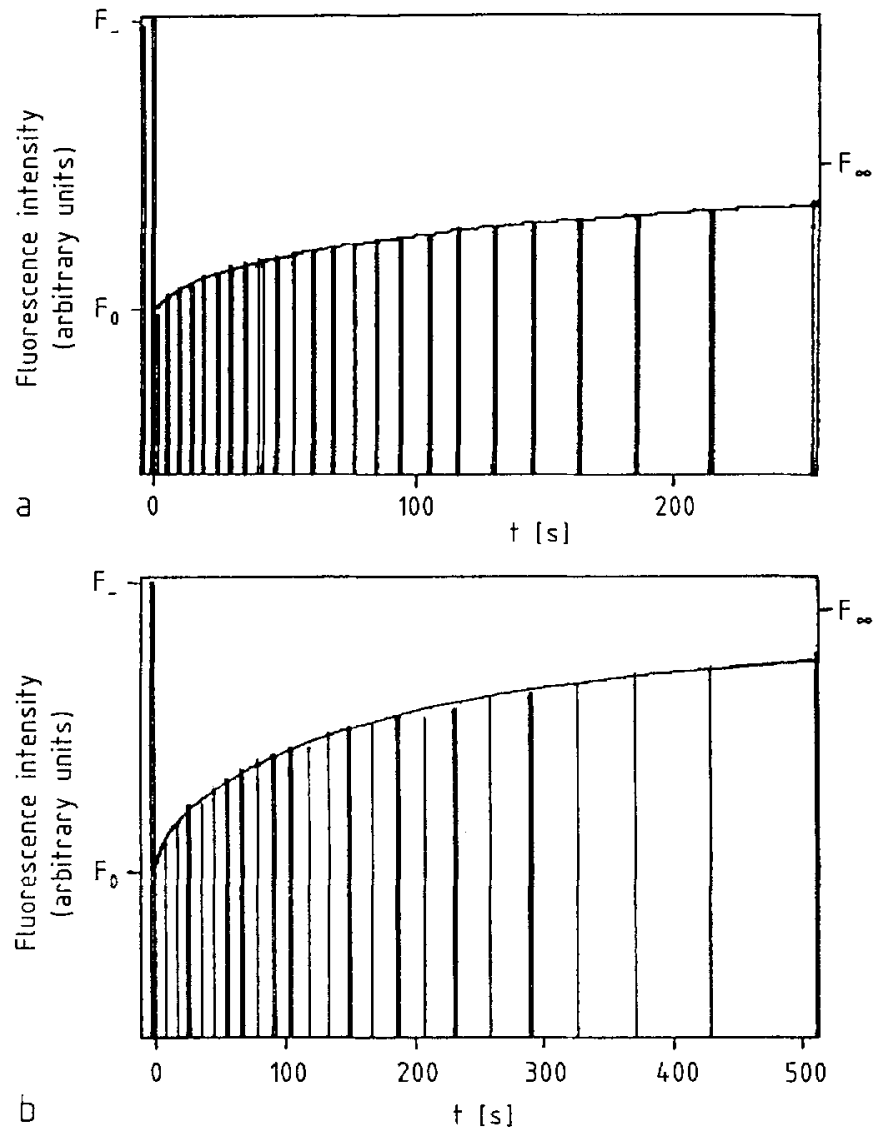

Fig. 1 a, b. Fluorescence-recovery curves for label Id (long-chain fatty acid) in the hydrophilic (a) and the hydrophobic (b) face of the cuticle of Clivia at $50^{\circ} \mathrm{C}$. The bars indicate experimental data (four measurements averaged), the lines represent best fits calculated according to Soumpasis (1983). The value $F_{-}$, as well as the fitted values $F_{0}$ and $F_{\infty}$ defined in eqn. 1 are marked. Note the different recoveries: a $R=51 \%$; b $R=91 \%$, indicating different mobile fractions

culars or a SIT camera (C1000 Type 12; Hamamatsu Photonics, Herrsching, FRG).

Only the central part of the laser beam was focussed onto the sample by placing an aperture of $0.15 \mathrm{~mm}$ inner diameter in the intermediate-image plane of the objective. In this way one achieves a rectangular intensity profile of the bleaching beam, which simplifies the evaluation of the fluorescence recovery curves (Axelrod et al. 1976).

For measurement of the fluorescence-recovery curves, the bleaching beam was switched on for $0.1-0.5 \mathrm{~s}$, which is short compared to the half-time of the fluorescence recovery. In order to avoid photobleaching during the measurement, the fluorescence was recorded intermittently at time intervals of $\Delta t_{0 b}=1 \mathrm{~s}$. The time intervals between these measurements increased with increasing observation time as shown in the examples of Fig. 1.

The fluorescence recovery is described in the conventional way (Axelrod 1976; Soumpasis 1983)

$F(t)=F_{0}+\left(F_{-}-F_{o o}\right) f(t)$

where $F_{0}$ is the intensity immediately after bleaching, $F_{-}$that before bleaching (see Fig. 1), and $F_{\infty}$ that at infinite time after bleaching, which is in practice not measurable. The function $f(t)$ equals zero for $t=0$ and is obtained by solving the two-dimensional diffusion equation. Following Soumpasis (1983), $f(t)$ can be expressed as

$f(t)=\left[I_{0}\left(\frac{2 \tau_{d}}{t}\right)+I_{1}\left(\frac{2 \tau_{d}}{t}\right)\right] e^{-\frac{2 \tau_{d}}{t}}$ 
where $I_{0}$ and $I_{1}$ are modified Bessel functions (Olver 1965). The so-called characteristic relaxation time $t_{d}$ is obtained by fitting the theoretical curves of Eq. 2 to the experimental $F(t)$-curves. The diffusion coefficient $D$ of the probe is then directly obtained according to

$D=\frac{w^{2}}{4 t_{c}}$

where $w$ is the radius of the bleaching spot.

The second important parameter obtained from the fluorescence recovery curves is the so-called fluorescence recovery

$R=\frac{F_{\infty}-F_{0}}{F_{-}-F_{0}}$

which is a measure for the fraction of probe molecules which are mobile.

The theoretical function $f(t)$ is fitted to experimental data $F(t)$ by variation of the parameters $F_{0}, F_{\infty}$ and $t_{d}$ in iterative procedure. If this procedure is applied to a hypothetical recovery curve without noise - obtained by computer simulation - the values of $D$ and $R$ are exactly equal to the corresponding input parameters. However, if the same statistical noise as observed in the experimental recovery curves is introduced, the values obtained by data-fitting deviate from the input parameters by $10 \%-50 \%$. For this reason, it is not meaningful to analyse recovery curves in terms of more than one recovery time unless the smallest and the largest time $t_{d}$ differ by more than a factor of 10 .

Scanning electron microscopy. Small pieces torn from the cuticles were stored in water and dried just before preparation. The samples were glued onto electron-microscopy grids and rotation-shadowed with platinum (1-5 nm thickness) at an angle of $45^{\circ}$. In some cases the cuticles were etched by ion bombardment or by suspension in a $14 \%$ (by vol.) solution of $\mathrm{BF}_{3}$ in methanol.

\section{Results}

Spin-label studies. The ESR spectra of stearic-acid probes labelled on either the 4-C atom (4-SASL) or the 14-C atom (14-SASL) of the chain were recorded from the intact cuticle, from the extracted waxes, and from the MX-membrane. The ESR spectra of the 14-SASL spin label in the intact cuticle (Fig. 2a) and in the extracted monomeric waxes (Fig. 2 b) were recorded over a wide range of temperatures, covering the likely physiological range. On hot sunny days, the adaxial leaf surface can be heated up to $45^{\circ} \mathrm{C}$, because of low heat conductivity of the cuticle. A further increase in temperature (over $45^{\circ} \mathrm{C}$ ) was of interest in order to study phase behaviour.

At low temperature $\left(10^{\circ}-20^{\circ} \mathrm{C}\right)$, the spectra are characteristic of chains whose rotational motion is strongly restricted on the conventional nitroxide ESR timescale and display hyperfine splittings which are close to the maximum possible. With increasing temperature, the spectral changes are at first relatively small, as is expected for spin labels in the slow motional regime. For the extracted waxes (Fig. 2b), the spectra then go through a series of complex spectral changes with further increase in temperature that are associated with the transition to the motional narrowing spectral regime, and possibly also with a broad region of lipid-phase separation. At high temperatures (over $>40^{\circ} \mathrm{C}$ ), the spectra from the waxes are motionally narrowed and character-
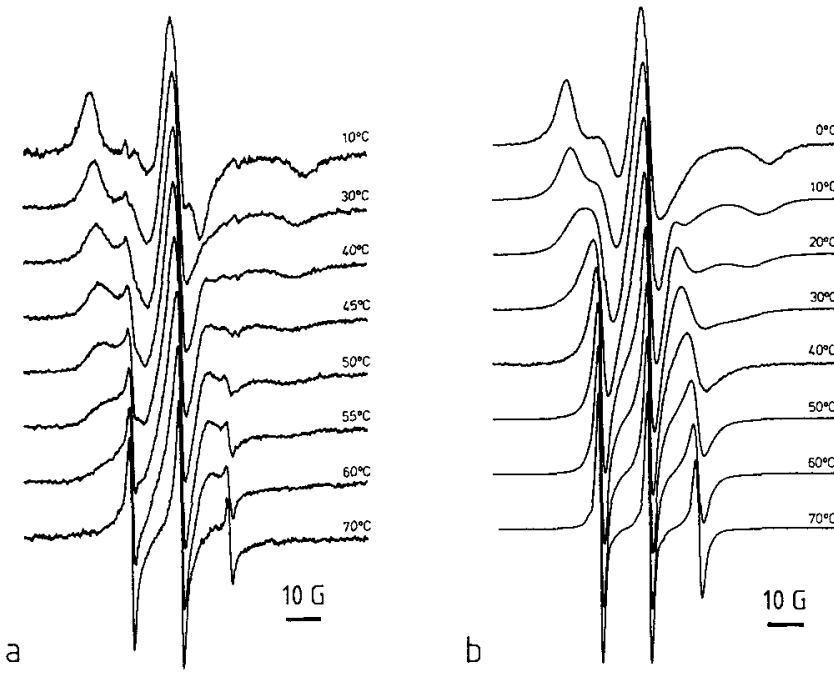

Fig. 2a, b. Electron-spin-resonance spectra as a function of temperature for the 14-SASL stearic-acid spin label in unoriented samples of the intact cuticle (a) and of the waxes extracted from the cuticle (b). Total scan range $=100$ gauss

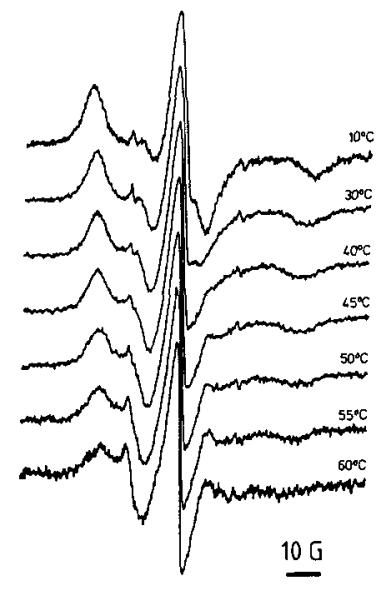

a

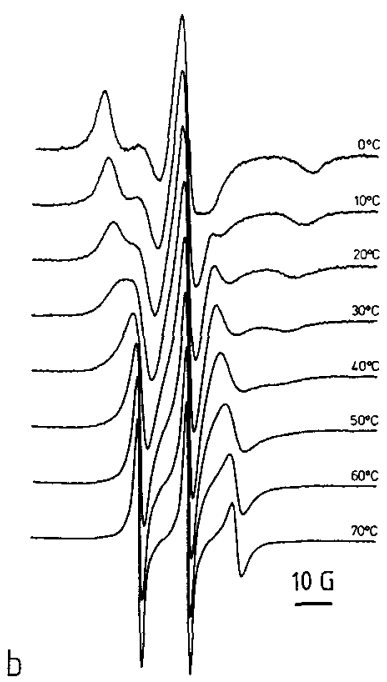

Fig. 3a, b. Electron-spin-resonance spectra as a function of temperature for the 4-SASL stearic-acid spin label in unoriented samples of the intact cuticle (a) and of the monomeric waxes extracted from the cuticle (b). Total scan range $=100$ gauss

istic of a near-isotropic rotational diffusion. The spectra from the intact cuticle (Fig. 2a) are even more complicated. At higher temperatures, they are clearly composed of two spectral components, one of which is similar to the motionally narrowed spectrum from the waxes, and the other of which is considerably broader and characteristic of a lipid-chain environment for which the rotational motions are still in the slow regime.

The ESR spectra of the 4-position stearic acid spin label (4-SASL) in the intact cuticle and in the extracted monomeric waxes (Fig. $3 \mathrm{a}, \mathrm{b}$ ) are qualitatively similar to the equivalent spectra from the 14-SASL label, although one label position corresponds to a chain segment close to the polar end of the fatty acid and the 


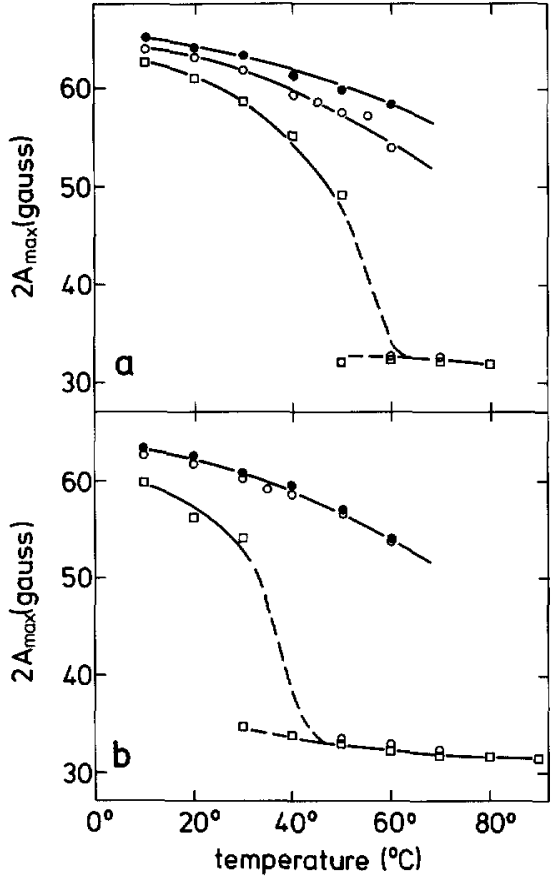

Fig. 4 a, b. Outer hyperfine splitting, $2 A_{\max }$, as a function of temperature for the 4-SASL stearic-acid spin label (a), and for the 14-SASL stearic-acid spin label (b), in intact cuticle (O), extracted waxes (D), and the polymer matrix (MX-membrane) from which the waxes hve been extracted ( $\bullet$. Where appropriate, values of $2 A_{\max }$ for both apparent co-existing spectral components are included

other to a segment close to the hydrophobic terminal methyl end of the chain. Quantitative differences can be seen between the temperature dependences for the two labels in that, at a given temperature, the mobility recorded at the 14-C atom segment is somewhat greater than that recorded at the 4-C atom segment.

The outer hyperfine splitting, $2 A_{\max }$, is given as a function of temperature in Fig. $4 \mathrm{a}, \mathrm{b}$ for spectra from the 4-SASL and 14-SASL spin labels, respectively, in the intact cuticle, in the extracted monomeric waxes, and in the MX-membrane. In the slow and intermediate motional regimes (rotational correlation time $>10^{-9} \mathrm{~s}$ ), the outer hyperfine splitting depends on the rate of motion of the labelled chain segment, and in the case of ordered chains also on the angular amplitude of the segmental motion (see e.g. Marsh 1986; Marsh and Horváth 1989). In the fast motional regime (rotational correlation time $\leqq 10^{-9} \mathrm{~s}$ ), the outer hyperfine splitting depends only on the amplitide of motion, and for isotropic motion assumes its minimum value.

For both the 4-SASL and 14-SASL labels, at low temperatures the values of $2 A_{\max }$ in the intact cuticle are rather similar to those in the polymer matrix, and are close to the maximum possible values (approx. 65 gauss), which correspond to slow motion on the conventional ESR timescale (rotational correlation time $\approx 10^{-8} \mathrm{~s}$ ). In the extracted waxes, the spectra at low temperatures also lie in the slow motional regime, but have appreciably smaller values of $2 A_{\max }$, corresponding to a somewhat greater motion than in the polymer matrix.
Table 1. Transition temperatures of crystals of monomeric waxes The values of the onsets $\left(T_{u}\right)$ and endpoints $\left(T_{l}\right)$ of the transitions as observed at decreasing temperature are given both for the label II a (4-SASL) and II b (14-SASL). The wax components were separated by thin-layer chromatography in a separate experiment (Hugues 1988). Some of the components could not be assigned $(+)$

\begin{tabular}{|c|c|c|c|c|c|}
\hline \multirow[b]{3}{*}{ Probe } & \multicolumn{5}{|c|}{ Phase transition temperatures in ${ }^{\circ} \mathrm{C}$} \\
\hline & \multicolumn{2}{|l|}{ II a } & \multicolumn{2}{|c|}{ IIb } & \multirow[b]{2}{*}{ Structure } \\
\hline & $T_{1}$ & $T_{n}$ & $T_{1}$ & $T_{u}$ & \\
\hline Mixture & 40 & 60 & 20 & 30 & \\
\hline Component 1 & 150 & & 90 & 130 & Alkanes \\
\hline Component 2 & 110 & & 30 & 110 & + \\
\hline Component 6 & 10 & 50 & 0 & 30 & Secondary alcohols \\
\hline Component 8 & 30 & 50 & 10 & 30 & + \\
\hline Component 9 & 40 & 50 & 20 & 50 & Fatty acids \\
\hline
\end{tabular}

At higher temperatures, the values of $2 A_{\max }$ for the MX-membrane decrease relatively slowly with increasing temperature, indicating that the spectra remain in the slow motional regime. For the extracted waxes, the values of $2 A_{\max }$ decrease more rapidly with increasing temperature, and then gradually convert to a motionally narrowed spectrum with a much smaller value of $2 A_{\max }$ that is characteristic of near-isotropic motion for both labels. This is a gradual change which does not correspond to an abrupt phase transition, as is evidenced also by continuous temperature scans of the central ESR line height which exhibits no discontinuities (compare Marsh and Watts 1981). In the intact cuticle, two spectral components are evident at the higher temperatures, one of which has values of $2 A_{\max }$ that are close to the nearisotropic value found for the monomeric waxes at higher temperatures.

On the whole, the peak separations for the 4-SASL and 14-SASL positional isomers of the stearic-acid spin label are rather similar, especially in the intact cuticle in the slow motional regime at lower temperatures and in the near-isotropic motionally narrowed regime for both the extracted waxes and in the intact cuticle at the higher temperatures. Appreciable differences are found between the hyperfine splittings of the two labels in the polymer matrix and in the waxes at the lower temperatures. These differences correspond to a greater degree of motion for the stearic acid labelled on the 14-C atom position (14-SASL).

Experiments with oriented samples of the native cuticle showed that the spectrum did not change with the orientation of the magnetic field relative to the cuticular surface, for both stearic-acid spin labels and throughout the whole temperature range studied (Figs. 2-4). The spectra from the oriented samples were essentially identical to those from the unoriented samples. No orientational dependence was observed either in the spectra from samples of the extracted monomeric waxes which were deposited from organic solution on the flat surface of the quartz tissue cell. The spectra were again identical to those from the unoriented samples. 

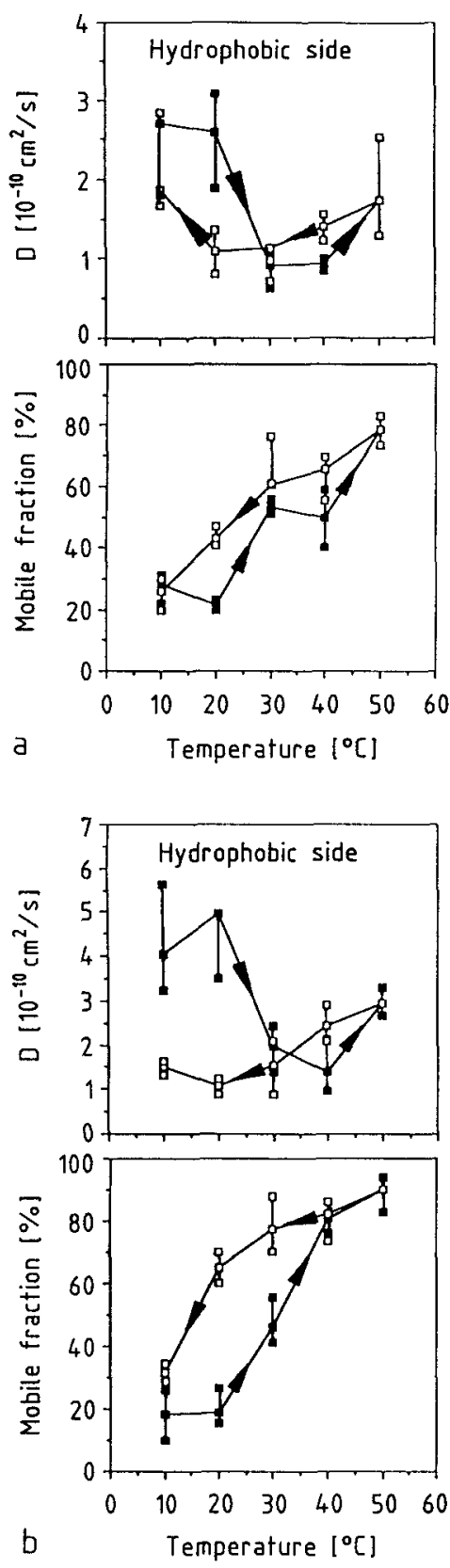
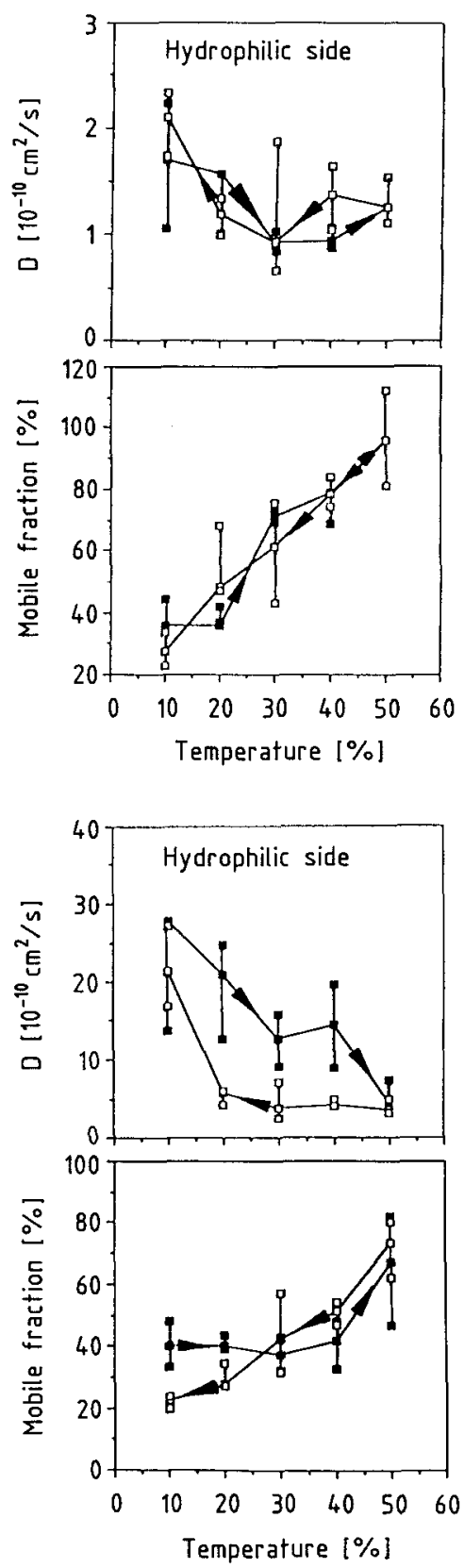

Fig. 5a, b. Lateral-diffusion coefficient, $D$, and mobile fraction, $R$, (as percent of total label content) of NBD-labelled alkane (structure Ia) for the intact (a) and for the wax-depleted (MX) cuticle (b). The data are given both for incubation of the hydrophobic (left side) and the hydrophilic (right side) face of the cuticle. Values were obtained for increasing ( $\square$ ) and decreasing ( $\square$ ) temperatures. The average values are interconnected by lines. The smallest and the largest values measured for $D$ and $R$ are also given and are connected to the averages by vertical bars
Using the present spin-label technique, the phase changes of several components of the monomeric waxes were studied systematically (Hugues 1988). Some results are given in Table 1.

Lateral diffusion of probes in both faces of the cuticular leaflet and wax-depleted cuticle. In the following figures (Figs. 5-7) we present measurements of the lateral-diffusion coefficient, $D$, and the mobile fraction, $R$, of fluorescent reporter molecules of various hydrophobicities (or polarities) as a function of temperature (between $10^{\circ} \mathrm{C}$ and $50^{\circ} \mathrm{C}$ ). The following points concerning the measurement procedure have been taken into account: (i) The cuticles were incubated with fluorescent probes at room temperature and measurements taken after first increasing the temperature stepwise (from $10^{\circ} \mathrm{C}$ to $50^{\circ} \mathrm{C}$ ) and subsequently after decreasing it. (ii) In some cases a second series of measurements was performed, again at increasing temperatures. The measured values agreed well with those of the descending curve even when the cuticles were annealed for some $2 \mathrm{~h}$ at room temperature. Thus the hysteresis found in some cases is attributed to the fact that probe molecules can penetrate all parts of the heterogeneously organized membrane only at a high temperature. (iii) For each temperature, $D$ and $R$ were measured at least three times at different positions and the average values are given. In some cases the largest and smallest values measured are also given to illustrate the errors of measurement caused by photon noise and inhomogenieties of the cuticle. The standard deviation could not be estimated because too few measurement points were recorded, and because of large statisti- 

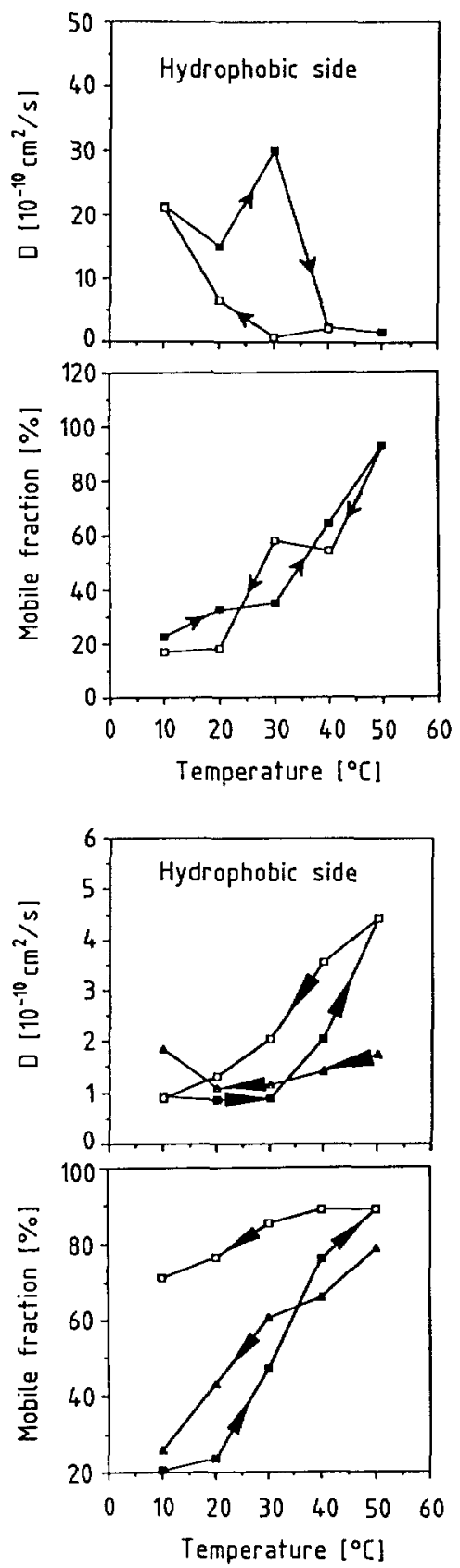
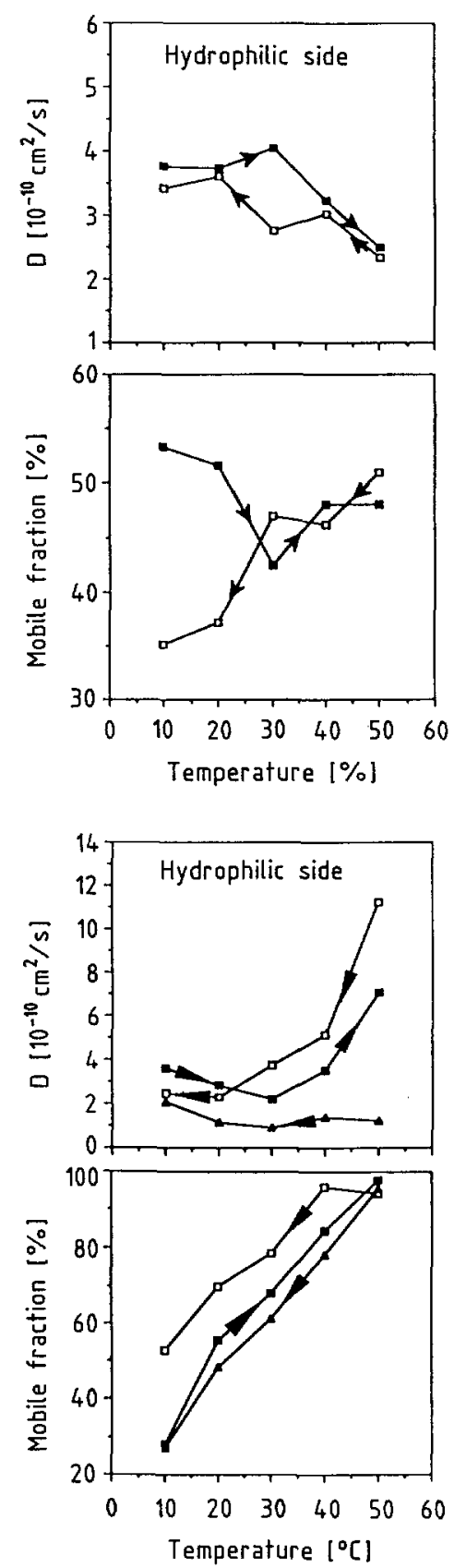

Fig. 6. Diffusion coefficient, $D$, and percentage of recovery, $R$, of a long-chain fatty acid (structure Ic) in the hydrophobic (left side) and hydrophilic (right side) faces of the intact cuticle of Clivia. All values measured are given. The solid straight lines interconnect the average values of $D$ and $R$ for ascending ( $\square$ ) and descending ( $\square$ ) temperatures
Fig. 7. Effect of growth of Clivia in the presence of ozone $\left(300-800 \mu \mathrm{g} \cdot \mathrm{m}^{-3}\right)$ on diffusion the coefficient (top curves) and mobile fraction (bottom curves) of NBD-labeled alkane. As before the cuticular leaflets were incubated on either the hydrophobic (left) or the hydrophilic faces (right). $\square$ Measurements for increasing temperature; ( $\square$ ) data for decreasing temperature. For comparison, the corresponding values of $D$ and $R$ for the normal plant obtained at decreasing temperatures are included $(\Delta)$. Statistical variation similar to Figs. 5 and 6 cal errors. (iv) The percentage of immobile molecules (that is $100-\mathrm{R}$ ) comprises all labels with a diffusion coefficient smaller than $D=10^{-11} \mathrm{~cm}^{2} \cdot \mathrm{s}^{-1}$. However, those molecules which are highly mobile but are confined within domains with extensions small compared to the bleaching spot diameter (that is $6 \mu \mathrm{m}$ in the present case) belong also to the immobile fraction defined as above. Thus $R$ gives valuable information about the heterogeneous organization of the cuticle.

Figure 5 shows the coefficient of lateral diffusion, $D$, and the mobile fraction, $R$, (as percent of total label concentration) for the most hydrophobic label studied: the NBD-labelled alkane (structure Ia). The data are given for both faces of the cuticular leaflet and for the wax-depleted cuticle. Even at $10^{\circ} \mathrm{C}$ a substantial fraction $(20 \%)$ of the molecules are highly mobile with the same or an even higher diffusion coefficient than at $50^{\circ} \mathrm{C}$. In many cases, $D$ first decreases (in an anomalous way) with increasing temperature (from $10^{\circ}$ to $30^{\circ} \mathrm{C}$ ) and increases only above $40^{\circ} \mathrm{C}$. The break at $40^{\circ} \mathrm{C}$ separates an anomalous from a normal temperature regime and indicates that a conformational change occurs between $40^{\circ} \mathrm{C}$ and $50^{\circ} \mathrm{C}$. Removal of the monomeric waxes does not appreciably affect the mobility in the hydrophobic face but the value of $D$ in the hydrophilic side increases by an order of magnitude at $10^{\circ} \mathrm{C}$ and by a factor of three at $50^{\circ} \mathrm{C}$.

The mobility and mobile fraction $R$ of the long-chain fatty acid (Ic) in both faces of the cuticular leaflet are shown in Fig. 6. As judged from the temperature dependence of the recovery the hydrophobic side exhibits two conformational changes: one between $20^{\circ} \mathrm{C}$ and $30^{\circ} \mathrm{C}$, 

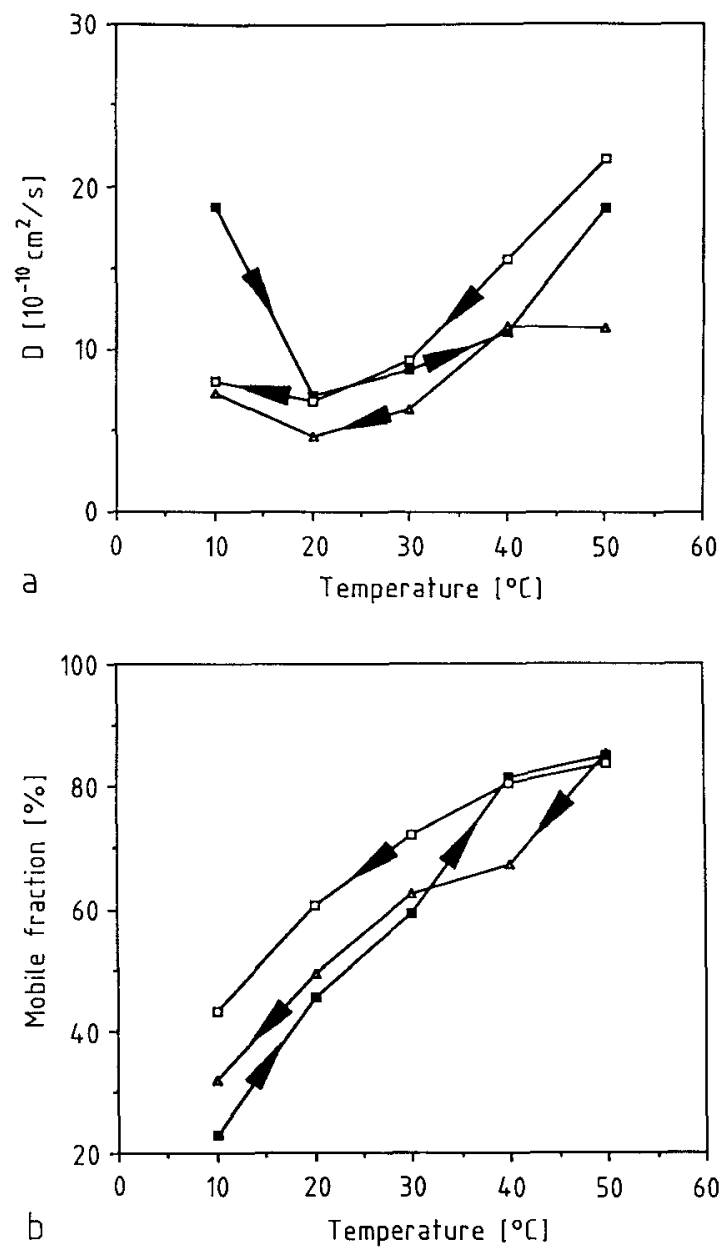

Fig. 8. Diffusion coefficient, $D$ (top), and mobile fraction, $R$ (bottom), of short-chain fatty acid (Id) in hydrophilic side of cuticular leaflet for plant grown in the presence of $300-800 \mu \mathrm{g} \cdot \mathrm{m}^{-3}$ ozone. Measurements at increasing ( $\square$ ) and decreasing $(\square)$ temperature

and one at about $45^{\circ} \mathrm{C}$. At the former, $R$ increases from $20 \%$ to $60 \%$ (regime I) and at the second (regime II) from $60 \%$ to $100 \%$. Rather astonishing is the very high diffusivity of the mobile fraction below $20^{\circ} \mathrm{C}(D$ $=21 \cdot 10^{-10} \pm 5 \cdot 10^{-10} \mathrm{~cm}^{2} \cdot \mathrm{s}^{-1}$ ) as compared to that at $50^{\circ} \mathrm{C}\left(1.7 \cdot 10^{-10} \pm 0.3 \cdot 10^{-10} \mathrm{~cm}^{2} \cdot \mathrm{s}^{-1}\right)$. The hydrophilic side again exhibits a conformational change between $20^{\circ} \mathrm{C}$ and $30^{\circ} \mathrm{C}$. However, in contrast to the hydrophobic side the recovery reaches only $50 \%$ at $50^{\circ} \mathrm{C}$ showing that a large fraction of the fatty acid is caged.

Effect of ozone on temperature dependence of mobility and mobile fraction. The effect of ozone which was added to the atmosphere at a concentration of $300-800 \mu \mathrm{g} \cdot \mathrm{m}^{-3}$ during growth of the plants is presented in Figs. 7 and 8 for the hydrophobic NBD alkane (Ia) and the highly hydrophilic short-chain fatty-acid probe (Id), respectively. The changes of the mobility compared to cuticles grown under normal conditions thus reflect modifications of the cuticular structure caused by perturbations arising during the development of the plant.

Figure 7 shows the result for the hydrophobic probe. For comparison the values of $D$ and $R$ obtained for a plant grown under normal conditions at decreasing temperature are also included. For both sides the lateral diffusion of probe I a for the ozone treated plant is much higher at $50^{\circ} \mathrm{C}$, i.e. by a factor of 2 in the cuticle proper and by a factor of 10 in the internal cuticular layer than in the control plant. The increase is somewhat smaller at $10^{\circ} \mathrm{C}$. The conformational change observed at about $20^{\circ} \mathrm{C}$ for the normal cuticle is less pronounced and appears to be shifted to higher temperatures by about $10^{\circ} \mathrm{C}$.

Figure 8 shows the effect of ozone of the diffusion coefficient, $D$, and recovery, $R$, for the highly hydrophilic short-chain fatty acid (Id). This probe can only be incorporated into the hydrophilic side. Both the diffusion coefficient and the mobile fraction are increased remarkably although considerably less than for the hydrophobic probe. This indicates that ozone effects primarily the structure of the hydrophobic layers of the cuticle. The same comparison was made for the long-chain fatty acid (data not shown). In this case the effect of ozone was within the limit of experimental error. This shows that the observed effect of ozone is not due to a systematic error.

\section{Discussion}

The cuticle of Clivia miniata belongs to the class of cuticles (such as that of Agave) with a well-ordered multilamellar leaflet at the outside (the so called cuticle proper $=\mathrm{CP}$ ) and two more disordered inner layers: the external (ECL) and the internal cuticular layer (ICL) (Wattendorf and Holloway 1982). However, as judged from negative-staining electron microscopy, all three layers appear to be made-up of multilamellar stacks, consisting of monolayers of well-defined thickness of $5 \mathrm{~nm}$ which are separated by more disordered layers of variable thickness (4-15 nm; Schmidt and Schönherr 1982; Riederer and Schönherr 1988).

The $5 \mathrm{~nm}$-layer could be a monolayer or a bilayer. For an average length of the polymerizable components of 18 carbon atoms, the thickness of the tightly packed layer would correspond to 2.5 monolayers. Such a layer could be formed by mutual shift of the chains in the normal direction. This would allow the formation of cross-links between the functional groups in the centre (e.g. secondary alcohols) and the ends (primary alcohols, carboxyl groups) of the wax molecules (Holloway $1980 \mathrm{~b}$ ). The two layers are clearly distinguished in negative-staining electron microscopy owing to the fact that the tight layers do not take up negative stain (such as $\mathrm{KMnO}_{4}$ ) whereas the disordered layers do so (Wattendorf and Holloway 1982; Schmidt and Schönherr 1982). In the outermost layer, the cuticle proper, the multilamellae are highly ordered and extend over the whole surface of the leaf, with the exception of certain cracks and defects (see Fig. 9a). In the ECL and the ICL, however, they are split-up into small domains of about $100 \mathrm{~nm}$ lateral extension.

Judged from negative staining experiments, the $\mathrm{CP}$ and ECL are at the average much more hydrophobic 

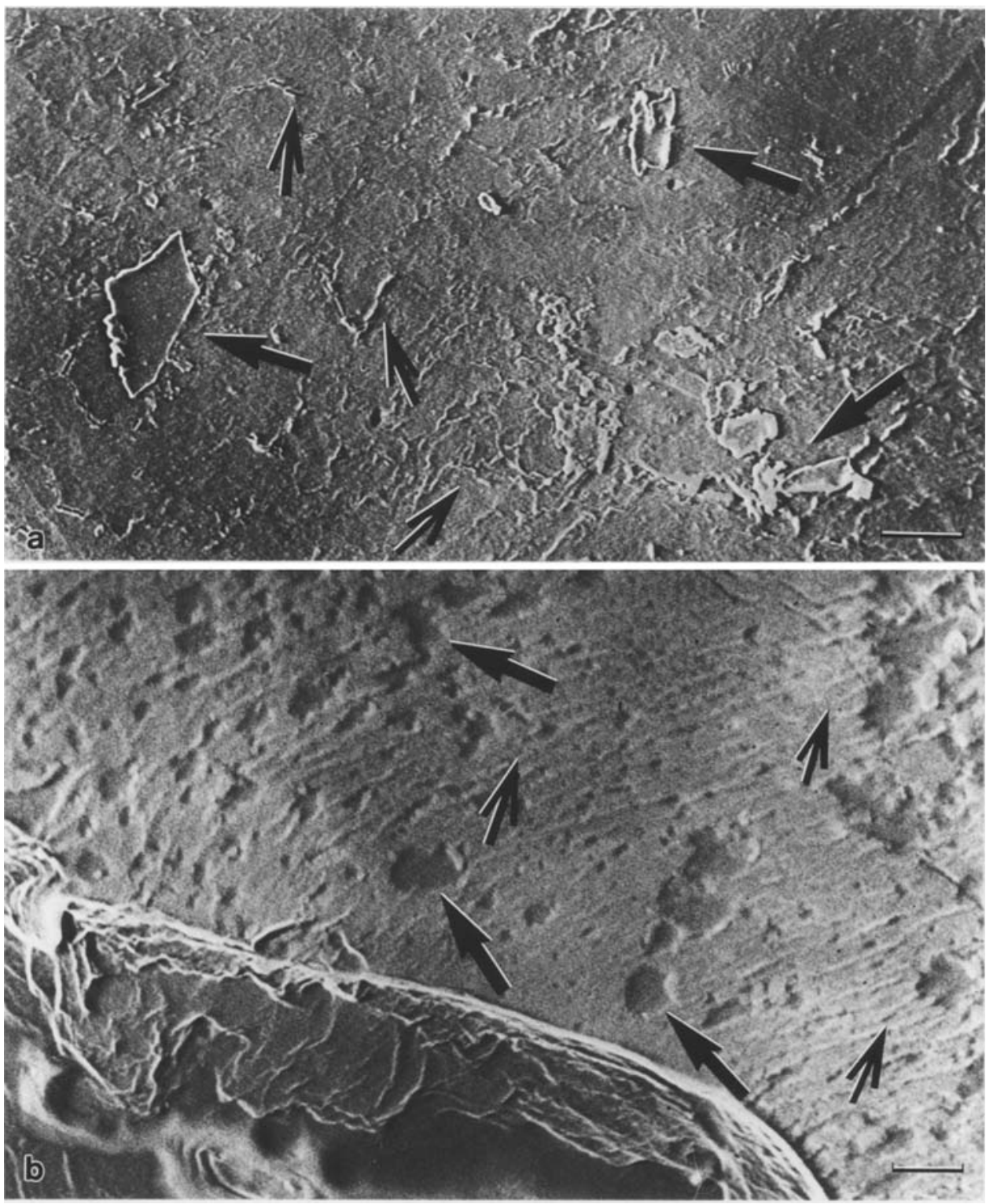

Fig. 9a, b. Scanning electron microscopy of Clivia miniata. (a) Scanning EM of surface of cuticle cut-out $22 \mathrm{~cm}$ above base of leaves. The small platelets indicated by thick arrows are polymerized cutin since they cannot be dissolved by organic solvents. Note also the rather large number of little cracks (thin arrows) which may be attributed to interfaces between two polymerized domains. Cuticles cut-our near the leaf base are smoother (J. Schönherr, personal communication). This means, the younger parts of the leaf show fewer defects. Bar $=10 \mu \mathrm{m}$. (b) Scanning EM of cuticular leaflet (cut-out $22 \mathrm{~cm}$ above base) which has been treated for $18 \mathrm{~h}$ with $\mathrm{BF}_{3}$ at $70^{\circ} \mathrm{C}$. Note the creation of holes (thick arrows) and wrinkles (thin arrows). The holes are most probably formed at the sites of cracks or defects in the outer layer, which are visible in $\mathbf{a}$. Bar $=1 \mu \mathrm{m}$ than the inner cuticular layer (ICL). This gradient in hydrophobicity is clearly demonstrated by the present finding that the short chain fatty acid can only be incorporated into the inner face of the cuticular layer.

According to Riederer and Schönherr (1988) the Clivia cuticles contain large amounts of epoxy fatty acids (such as Agave leaves) which can form cross-links not degradable by $\mathrm{BF}_{3}$. These bonds are mainly located in the two outer (hydrophobic) layers: the ECL and the $\mathrm{CP}$, and most probably formed within the $5 \mathrm{~nm}$ thick, tight layers. This is also suggested by Fig. $9 \mathrm{~b}$. Firstly, it shows that only small holes are created in these layers by treatment with $\mathrm{BF}_{3}$ for $18 \mathrm{~h}$ at $70^{\circ} \mathrm{C}$. Secondly, the layers assume a wrinkled surface profile which suggests that the more hydrophilic, amourphous material between the $5 \mathrm{~nm}$-layers is at least partially degraded. The surface of the cuticle exhibits little cracks (cf. arrows in Fig. 9a) at which $\mathrm{BF}_{3}$ degradation of the cuticular matrix could start. The above mentioned experimental observa- tions suggest the model of the multilamellar stacks as presented in Fig. 10.

The monomeric waxes amount to only a few percent of the total cuticular mass of Clivia cuticle. The fact that removal of these waxes does not affect the microstructure of the cuticle indicates that these are largely randomly distributed within the cuticular matrix and do not form larger crystalline aggregates. Further evidence for this conclusion comes from our finding that the conformational transition of the intact cuticle and the extracted waxes occure at markedly different temperatures (cf. Fig. 5). This may be completely different in other plant cuticles where distinct changes in the birefringence are observed and the outer layer may even collapse after removal of the waxes (Sitte and Rennier 1963).

The diffusion coefficients are presented in Table 2 for the lowest $\left(10^{\circ} \mathrm{C}\right)$ and the highest $\left(50^{\circ} \mathrm{C}\right)$ measuring temperature. In addition a value for an intrinsic fluorescent component (most probably a phenolic species) 
Table 2. Summary of diffusion coefficients, $D$, in cuticle of Clivia miniata of NBD-labelled molecules of increasing hydrophilicity (from left to right). The values of $D$ at $10^{\circ} \mathrm{C}$ and $50^{\circ} \mathrm{C}$ as measured at decreasing temperature are given. For some probes, the values for normal and ozone treated plants are compared

Probe \begin{tabular}{llllll}
$D\left[10^{-10} \mathrm{~cm}^{2} \cdot \mathrm{s}^{-1}\right]$ & & & & \\
\hline Ia & Ib & Ic & Id & Intrinsic
\end{tabular}

Normal cuticle, hydrophobic side

$\begin{array}{cccccc}10^{\circ} \mathrm{C} & 1.9 \pm 0.5 & 39 \pm 10 & 21 \pm 5 & - & 20 \pm 5 \\ 50^{\circ} \mathrm{C} & 1.7 \pm 0.6 & 60 \pm 20 & 1.7 \pm 0.3 & - & 17.5 \pm 7\end{array}$

Ozone-treated cuticle, hydrophobic side

$\begin{array}{ccccccc}10^{\circ} \mathrm{C} & 1.0 \pm 0.4 & - & 20 \pm 2 & - & 44 & \pm 6 \\ 50^{\circ} \mathrm{C} & 4.4 \pm 0.8 & - & 1.6 \pm 0.3 & - & 21 & \pm 3\end{array}$

Normal cuticle, hydrophilic side

$\begin{array}{llllcl}10^{\circ} \mathrm{C} & 2.0 \pm 0.3 & 11 \pm 3 & 3.4 \pm 0.6 & 7.3 \pm 1.5 & - \\ 50^{\circ} \mathrm{C} & 1.2 \pm 0.2 & 28 \pm 10 & 2.4 \pm 0.6 & 10.5 \pm 2 & -\end{array}$

Ozone-treated cuticle, hydrophilic side

$\begin{array}{llllrl}10^{\circ} \mathrm{C} & 2.5 \pm 1 & 15 \pm 4 & 4.3 \pm 0.7 & 8.1 \pm 1 & - \\ 50^{\circ} \mathrm{C} & 9.1 \pm 4 & 26 \pm 5 & 2.2 \pm 0.6 & 20.1 \pm 4 & -\end{array}$

MX-membrane, hydrophobic side

$10^{\circ} \mathrm{C} \quad 1.5 \pm 0.2 \quad 16 \pm 6$

$50^{\circ} \mathrm{C} \quad 3.0 \pm 3 \quad 16 \pm 3$

MX-Membrane, hydrophilic side

$10^{\circ} \mathrm{C} \quad 21 \pm 5 \quad 8 \pm 1$

$50^{\circ} \mathrm{C} \quad 4.0 \pm 1 \quad 14 \pm 5$

probe is given. The data in Table 2 reflect the heterogeneous organization of the cuticle which renders an unambiguous interpretation of the results rather difficult. However, results from numerous diffusion studies in model membranes (Galla et al. 1979; Vaz et al. 1985), together with the model of Fig. 10, give the following important clues to an interpretation of the diffusion measurements: In a given homogeneous system, $D$ depends weakly on the size of the diffusant. Thus the large difference by an order of magnitude between the D-values of say the alkane (Ia) and the alcohol (Ib) is certainly due to the fact that these two probes reside and diffuse primarily in different environments. The smallness of $D$ for Ia suggests that this highly hydrophobic probe is mainly dissolved in the dense $5 \mathrm{~nm}$-layers whereas the alcohol resides more in the hydrophilic, disordered layers.

As is well known (Galla et al. 1979) that $D$ decreases exponentially with the packing density of the solvent as suggested by the free-volume model of diffusion. This rule could explain our finding that the $D$-value for the probe Ia and in the ICL increases by a factor of about five after removal of the monomeric waxes, which is expected to decrease the lateral packing density in the monolayers.

An important result is the finding of a large fraction (of more than $20 \%$ ) of mobile molecules at $10^{\circ} \mathrm{C}$, the

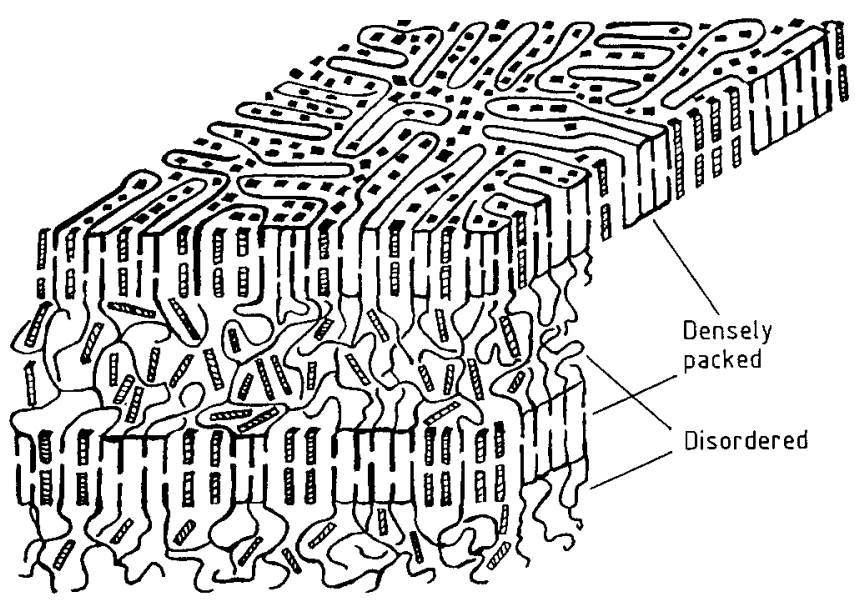

Fig. 10. Possible microscopic design of cuticles of Clivia miniata and Agave from ordered monolayers of wax molecules, which are separated by more disordered layers. The former have a well-defined thickness of $5 \mathrm{~nm}$ and are highly hydrophobic as follows from the finding that they are not stained by $\mathrm{KMnO}_{4}$. The latter vary in thickness between 4 and $15 \mathrm{~nm}$. In the outer layer - the cuticle proper - the lamellae extend over macroscopic dimensions (order of $\mathrm{cm}$ ) and the stacks of multilayers are only occasionally interrupted by dislocations. The two inner layers appear to be composed also largely of small domains of such alternating stacks. Their extension parallel to the planes of the layers is at most some $100 \mathrm{~nm}$ and the number of stacks is about 5 . The waxes act as conditioner which may for instance fill the gaps between interfaces of adjacent polymerized domains. To a large extent they determine the phase changes

diffusion coefficient of which is in some cases even higher than at $50^{\circ} \mathrm{C}$. This is explained in terms of lateral phase separation within the layers leading to the formation of a mosaic-like structure of fluid and rigidified domains. The fluid domains are expected to be formed by a lowmelting monomeric wax component. As indicated by data given in Table 1, some components indeed exhibit phase transitions at $T \approx 10^{\circ} \mathrm{C}$. Moreover, from the absence of the phase change in wax-depleted cuticles it follows that the monomers determine the phase-separation processes in the cuticles. Very large D-values are expected if the fluid domains from narrow paths, for instance at the interface between two adjacent platelets of polymerized cutin (see Fig. 10), and if these paths form an interconnected network over large extensions. The diffusion can then be described in terms of a percolation process (Saxton 1982; Gaub et al. 1984).

The temperature dependencies of both $D$ and $R$ strongly indicate that all three layers (ECL, ICL, CP) exhibit a conformational change at about $20^{\circ} \mathrm{C}$ which is associated with a continuous fluidization of the cuticle at increasing temperature. Since one is dealing with a multi-component system, this conformational change has to be interpreted in terms of the onset of the phase separation mentioned above. This agrees well with the abrupt decrease of the order parameter of the spin label $\mathrm{II} b$ at this temperature. The hydrophobic face of the 
cuticle (comprising the ECL and the $\mathrm{CP}$ according to Holloway 1980 a) exhibits an additional transition at a much higher temperature, i.e. at about $45^{\circ}$ to $50^{\circ} \mathrm{C}$. This is at least verified by the diffusion measurement of the long-fatty-acid NBD label (Id) since its mobile fraction increases abruptly above $40^{\circ} \mathrm{C}$. One possible explanation of this phase change is that it is associated with the melting within the tightly packed $5 \mathrm{~nm}$-layers. This is also suggested by the finding that the D-value for the alkane increases abruptly by a factor of two at $40^{\circ} \mathrm{C}$.

Comparison of the spectra from the 4-position and 14-position spin labels yields information regarding the dynamic chain ordering in the cuticular layers. It is only in the motional narrowing regime that it is possible to distinguish unambiguously between chain ordering and chain mobility. In this temperature region, both the 4SASL and the 14-SASL labels yield near-isotropic spectra. There is no indication for the presence of an axially symmetrical spectrum such as is characteristic for the chain-ordering in phospholipid bilayers or cell membranes. In addition, no angular dependence was found for the spectra from oriented samples of the intact cuticle. The latter is contrary to the proposals of Eckl and Gruler (1980) for the cuticle of Citrus aurantium L., but is fully consistent with the observation of a near-isotropic rotational motion.

In summary, the fatty acid ESR probes record the environments of both the polymerized matrix and the waxes in the intact cuticle. At higher temperatures, the chain mobility in the regions of monomeric wax is considerably greater than that in the polymerized matrix. For the waxes, the chain motion is not characteristic of a liquid crystalline structure such as is found in cell membranes (for instance the thylakoid membrane - see e.g. Gang et al. 1989), but exhibits a much more isotropic rotational diffusion both close to the polar group of the lipid chain and close to its hydrophobic terminal methyl region. Although the spectra from the polymerized regions do not give information on chain-ordering in the accessible temperature range, it is likely from the apposition with the regions of waxes that the motion of the polymerized chains is also of a more isotropic character, consistent with the lack of any orientation dependence in the spectra. The results are consistent with the function of the waxes as a binding material, ensuring the impermeability of the cuticular layer to water (Schönherr 1976), whereas the polymer matrix provides the necessary structural stability and organisation.

One aim of the present study was to elucidate the effect of ozone on the structural and dynamic properties of cuticles of leaves. We have clearly demonstrated that the presence of realistic amounts of ozone during growth facilitates molecular transport within the cuticle remarkably and may have indeed dramatic effects on the diffusion coefficient of some species. At the present stage of our knowledge an interpretation in terms of structural changes cannot be given. Our finding that ozone affects primarily the diffusion of hydrophobic molecules suggests, however, that it is mainly the hydrophobic part of the cuticle which is affected. One possibility is that ozone perturbs the polymerization within the tightly packed layers.

The present work was made possible by a grant from the Bayerische Umweltministerium. Additional support by the Fonds der Chemischen Industrie is gratefully acknowledged. We are most grateful for very helpful discussions with Professor $\mathrm{H}$. Ziegler, Professor J. Schönherr and Dr. M. Riederer from the Institut für Botanik, Technische Universität München, FRG.

\section{References}

Axelrod, D., Koppel, D.E., Schlessinger, J., Elson, E., Webb, W.W (1976) Mobility measurements by analysis of fluorescence photobleaching recovery kinetics. Biophys. J. 16, 1055-1069

Baker, E.A. (1980) The influence of environment on leaf wax development in Brassica Oletracea var. gemmifera. New Phytol. 73 955-966

Eckl, K., Gruler, H. (1980) Phase transitions in plant cuticles. Planta 150, $102-113$

Galla, H.J., Hartmann, W., Theilen, U., Sackmann, E. (1979) On two dimensional passive random walk in lipid bilayers and fluid pathways in biomembranes. J. Membr. Biol. 48, 215-236

Gang, L., Knowles, .F., Murphy, D., Nishida, I., Marsh, D. (1989) Spin label ESR sutdies of lipid-protein interactions in thylakoid membranes. Biochemistry, in press

Gaub, H., Sackmann, E., Büschl, R., Ringsdorf, H. (1984) Lateral diffusion and phase separation in two-dimensional solutions of polymerized butadiene lipid in dimyristoyl-phosphatidylcholine bilayers. Biophys. J. 45, 725-731

Hallam, N.D. (1980) Fine structure of the leaf cuticle and the origin of leaf waxes. In: The plant cuticle, pp. 197-214, Cutler, D.F., Alvin, K.L., Price, C.E., eds. Academic Press, London New York

Holloway, P.J. (1980a) Structure and histochemistry of plant cuticular membranes: an overview. In: The plant cuticle, pp. 1-32, Cutler, D.F., Alvin, K.L., Price, C.E., eds. Academic Press, London New York

Holloway, P.J. (1980b) The chemical constitution of plant cuticles. In: The plant cuticle, pp. 45-85, Cutler, D.F., Alvin, K.L., Price, C.E., eds. Academic Press, London New York

Hubbell, W.L., McConnell, H.M. (1971) Molecular motions in spinlabelled phospholipids and membranes. J. Am. Chem. Soc. 93, $314-326$

Hugues, B. (1988) Diploma Thesis. Technical University Munich, FRG

Juniper, B.E. (1957) The effect of pre-emergent treatment of peas with trichloracetic acid on the submicroscopic structure of the leaf surface. New Phytol. 58, 1-5

Kerler, F., Riederer, M., Schönherr, J. (1984) Non-electrolyte permeability of plant cuticles: A critical evaluation of experimental methods. Plant Physiol. 62, 599-602

Marsh, D. (1986) Spin label ESR spectroscopy and molecular mobility in biological systems. In: Supramolecular structure and function pp. 48-62, Pifat-Mrzljak, G., ed. Springer, Berlin Heidelberg New York

Marsh, D., Horváth, L.I. (1989) Spin label studies of the structure and dynamics of lipids and proteins in membranes. In: Advanced EPR in biology and biochemistry. Hoff, A.J., ed. Elsevier, Amsterdam, in press

Marsh, D., Watts, A. (1981) ESR spin label studies of liposomes. In: Liposomes from physical structure to therapeutic applications pp. 139-188, Knight, C.G., ed. Elsevier/North-Holland Biomedical Press, Amsterdam

Olver, F.W.J. (1965) Bessel functions of integer order. In: Handbook of mathematical functions, pp. 355-433, Abramowitz, M., Stegun, I.A., eds. Dover Publications, New York

Orgell, W.H. (1955) The isolation of plant cuticle with pectic enzymes. Plant Physiol. 30, 78-80 
Riederer, M., Schönherr, J. (1988) Development of plant cuticles: fine structure and cutin composition of Clivia miniata Reg. leaves. Planta 174, 127-138

Saxton, M.J. (1982) Lateral diffusion in an archipelago. Biophys. J. 39, 165-173

Schmidt, H.W., Schönherr, J. (1982) Development of plant cuticles: occurrence and role of non-ester bonds in cutin of Clivia miniata Reg. leaves. Planta 156, 380-384

Schönherr, J. (1976) Water permeability of isolated cuticular membranes: the effect of cuticular waxes on diffusion of water. Planta 131, $159-164$

Sitte, P., Rennier, R. (1963) Untersuchungen an cuticularen Zellwandschichten. Planta 60, $19-40$
Soumpasis, D.M. (1983) Theoretical analysis of fluorescence photobleaching recovery experiments. Biophys. J. 41, 95-97

Vaz, W., Clegg, R.M., Hallmann, D. (1985) Translational diffusion of lipids in crystalline phase of phosphatidyl choline multibilayers. A comparision of experiment with theory. Biochemistry 24, 781-786

Wattendorf, J., Holloway, P.J. (1982) Studies on the ultrastructure and histochemistry of plant cuticles: Isolated cuticular membrane preparations of Agave americana and effects of various extraction procedures. Ann. Bot. 49, 769-804

Received 23 August 1989; accepted 24 January 1990 